\title{
THE EFFECT OF CORONARY OCCLUSION UPON THE INITIAL PHASE OF THE VENTRICULAR COMPLEX IN PRECORDIAL LEADS
}

\author{
By SAMUEL BELLET ANd CHARLES G. JOHNSTON \\ (From the Robinette Foundation, the Department of Research Surgery, University of \\ Pennsylizania, and the Dirrision of Cardiology, Philadelphia General \\ Hospital, Philadelphia)
}

(Received for publication Februa:y 28, 1934)

In electrocardiograms of normal human hearts recorded by precordial leads the initial ventricular complex shows a fairly constant configuration. With the right arm electrode placed at the apical region and the second electrode at any point distant from the heart the primary ventricular deflection consists of a conspicuous downward deflection followed by an upward deflection. The initial downward deflection which is constantly present in normal controls entirely disappears or becomes conspicuously smaller after infarction involving the apex, lower anterior and lateral portions of the left ventricle ${ }^{1}$ (8) (10) (11). Moreover, the initial downward deflection is influenced by the position of the anterior electrode as well as by pathological changes within the heart, decreasing in amplitude and even disappearing as the electrode moves from the apex toward the base of the heart.

The electrocardiogram of the dog and the cat, obtained by precordial leads is similar to that observed in the human. Moreover, the influence of change of position of the anterior electrode resembles that occurring in man. Because of this similarity and because the use of animals allows the changes to be studied at frequent intervals from the very moment of muscle injury until repair and recovery have reached their maximum, we have used these animals to study the effects of myocardial infarction upon the initial downward deflection of precordial leads.

In general, the experiments fall into two groups (1) acute and (2) survival experiments. In the former, the effect of muscle injury produced by ligation of different coronary arteries, or the damage resulting from the application of a cautery directly to the heart muscle upon the initial downward deflection was observed over a period of about two hours. In the survival experiments, coronary arteries were ligated and the electrocardiographic change followed over a period of months.

The purpose of this paper is to record the results of experiments in dogs

\footnotetext{
${ }^{1}$ Occasionaily this deflection disappears when apical involvement alone is present.
} 
and cats designed to show the location and extent of myocardial lesions necessary either to modify or cause the disappearance of the initial downward deflection of precordial leads.

\section{ACLTE EXPERIMENTS}

\section{METHODS}

The animals were anesthetized by the administration intraperitoneally, of $50 \mathrm{mgm}$. of sodium amytal per kilogram of body weight. Artificial respiration was instituted by means of a tracheal cannula and a bellows. The electrodes were attached for taking indirect leads; additional electrodes were sutured beneath the skin of the anterior and posterior cardiac region so that precordial leads could also be obtained. Three precordial leads were routinely taken in our experiments, anteroposterior (Lead IV), anterior to left leg ( Lead V), and posterior to left leg (Lead VI). The right arm wire was attached to the anterior, and the left arm wire to the posterior, an arrangement which in the normal animal yielded an electrocardiogram having an initial downward deflection followed by an upward deflection.

The chest was opened by cutting the second, third, fourth and fifth ribs along the left posterior axillary line; the heart was exposed by opening the pericardium and the selected coronary arteries were ligated. The heart was then returned to its original position, the ribs and skin were approximated and electrocardiograms taken at frequent intervals. In some animals the pericardium was closed, in others it was not sutured; there was no difference in the resulting electrocardiograms. Control studies showed that merely opening and closing the chest in the manner described was without significant effect upon the electrocardiograms. In the acute experiments where the myocardium was injured by cauterization of selected regions of the heart instead of coronary artery ligation, this injury was accomplished by an electric cautery at red heat applied until the superficial portion of the myocardium was practically carbonized. At the end of both types of experiment, which usually lasted not more than two hours, the animals were sacrified.

\section{RESUI.TS}

The effects of the procedures just suggested upon the initial downward deflection of precordial leads were to some extent complicated; for clarity the result of each procedure will be presented separately.

\section{Ligation of branches of the coronary arteries}

Ligation of the anterior descending branch of the left coronary artery, performed in $12 \mathrm{dogs}$ and in 6 cats, produced consistent $\mathrm{R}-\mathrm{T}$ interval deviations of the type clescribed by Wood and Wolferth (14) but failed to alter the initial downward deflection.

Dogs:

Ligation in three dogs of the septal branch of the left coronary artery produced no change in the initial downward deflection.

Ligation in five logs of the anterior divisions of the circumflex branch of the left coronary artery was without influence upon the initial downward deflection. 
Ligation of the anterior descending plus the septal branch of the left coronary artery resulted in a slight diminution of the initial downward deflection in some experiments. In those experiments, where the anterior branches of the circumflex were ligated in addition to the two vessels of the previous experiments, the animals died soon afterward, usually within a few minutes after the last vessel was tied. Electrocardiograms taken during this brief period showed no change in the initial downward deflection.

\section{Cats:}

Ligation of single coronary arteries in dogs and the combinations above mentioned having failed to cause a disappearance of the initial downward deflection, ligation of similar vessels was next attempted in the cat. That cats can withstand multiple coronary artery ligations much better than dogs has been demonstrated to our satisfaction by numerous experiments. The heart of the cat can beat vigorously for long periods after ligation of one and often after ligation of the two main branches of the left coronary artery.

Ligation of the anterior descending branch in cats resulted in $\mathrm{R}-\mathrm{T}$ deviations, but without disappearance of the initial downward deflection in Lead IV of the precordial leads. Ligation in four cats of the anterior descending branch of the left coronary artery plus the anterior branch of the circumflex resulted in a complete disappearance of the initial downward deflection and an R-T interval deviation situated below the iso-electric line, the resulting electrocardiogram (Fig. 1) being practically identical with that observed in the human after acute occlusion of the anterior descending branch of the left coronary artery (11) (13).

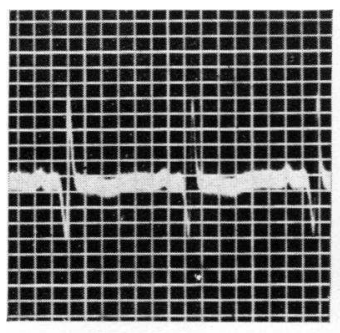

A

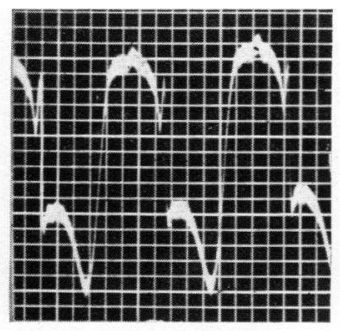

B

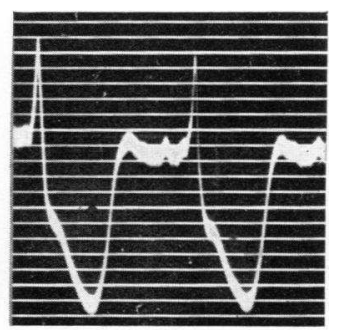

$\mathrm{C}$

Fig. 1. 1 Showing the Effect in Lead IV of Ligation of the Anterior Descending Branci of the Left Coronary Artery Plus the Anterior Branch of the Circumflex Artery in the Cat

$(A)$ Normal. (B) After ligation of the anterior descending branch of the left coronary artery. $(C)$ After ligation of the anterior descending and circumflex branches of the left coronary artery; note the absence of the initial downward deflection and S-T interval situated below the iso-electric line.

1 The initial phase of the ventricular complex. which shows clearly in the negatives, has been retouched for purposes of reproduction in this and subsequent figures. 


\section{Cauterization of the heart muscle}

Having observed the results of ligation of large coronary arteries, an endeavor was made to compare the results of cauterization of the heart muscle; first, because the area of damage brought about by this method may be accurately controlled, and secondly, because a slightly different type of muscle injury is produced.

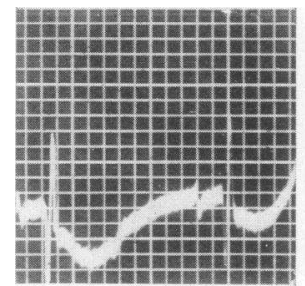

A

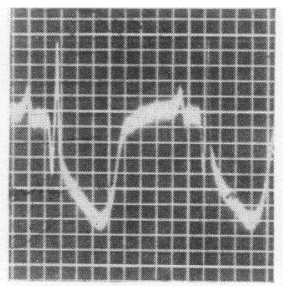

B

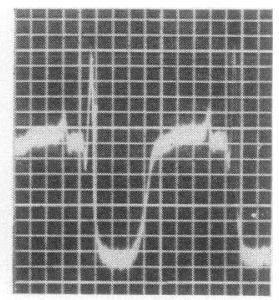

C

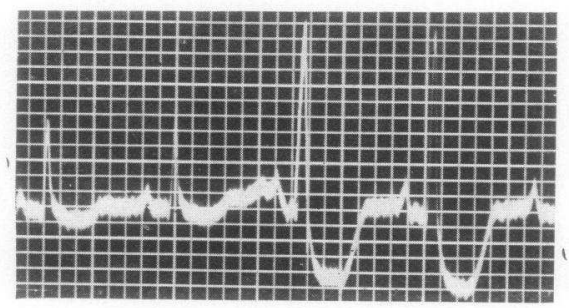

D

Fig. 2 (Experiment August 25, 1932). Showing the Effects in Lead IV of Cauterization of the Heart Muscle in Dogs

$(A),(B),(C),(D)$ show the effect of cauterization of the heart muscle. $(A)$ only small portion of apex cauterized, note the depression of $\mathrm{S}-\mathrm{T}$ interval; the initial downward deflection is little affected. (B) Area of cauterization increased to involve lower third of anterior wall of the left ventricle; note the increase in depression of the S-T interval. The initial downward deflection is slightly diminished in amplitude. (C) Area of cauterization increased to involve lower two-thirds of the anterior wall of left ventricle including the apex and a portion of right ventricle adjacent to interventricular groove. Note the almost complete absence of initial downward deflection and marked depression of the S-T interval. (D) Same as $C$. Note the variation in the ventricular complex with respiration, the largest complexes occur with inspiration, the smallest with expiration. Note the depression of S-T interval and the absence of the initial downward deflection.

In four experiments in dogs in which the apex, and the lower half of the anterior and lateral walls of the left ventricle were cauterized, the initial downward deflection disappeared in only one $\mathrm{dog}$; in the remaining three the initial downward deflection, while diminished, did not disappear. In three other experiments the entire anterior portion of the heart (both right 
and left ventricles) and the lateral portion of the left ventricle were cauterized before the initial or downward deflection disappeared (Fig. 2). In three other dogs where a similar portion of the heart was cauterized the initial downward deflection, while diminished did not disappear. Apparently the extent of the cauterized area is an important factor in determining the final results.

In cats no conspicuous change in the initial downward deflection was observed when the right ventricle alone was cauterized. The amplitude of this deflection was diminished most when the apex, and the lower anterior and lateral portions of the left ventricle were cauterized.

In three cats the initial downward deflection disappeared and an R-T deviation situated below the iso-electric line resulted after cauterization of the apex and the lower two-thirds of the anterior and lateral walls of the left ventricle, including a narrow strip of right ventricle adjacent to the anterior interventricular groove.

The ventricular complexes in these experiments as well as the others in which the chest had been opened, often showed a phasic variation with respiration, the highest amplitude being attained at inspiration, the lowest at expiration (Fig. 2D). The disappearance of the initial downward deflection was always judged from the complexes with the highest amplitude.

\section{Effect of injury of a small arca of heart muscle upon the initial downatard deflection of the clectrogram}

The purpose of this experiment was to compare the electrogram from an electrode placed directly on a small injured portion of the heart muscle with the tracings obtained from the precordial leads after such injury.

The heart was exposed and the animal arranged for taking electrograms as well as precordial leads. In leading off from the heart directly, electrodes soaked in copper sulphate contained in narrow glass tubes were used. An area about a centimeter and a half in diameter on the anterior surface of the left ventricle near the apex was selected and control electrograms taken. The right arm electrode was the one which led off from the heart directly, the left arm electrode being in contact with the chest posteriorly; the left leg electrode was sutured beneath the skin of the left leg as in previous experiments. That portion of the left ventricle in contact with the direct lead electrode was then cauterized and the electrogram repeated. A control was again taken from an uninjured portion of the heart on the anterior surface of the right ventricle. Needle electrodes were then inserted into the myocardium immediately beneath the cauterized area and electrograms again taken.

In the control tracings with the electrode placed directly upon the normal muscle of the left ventricle the configuration of the electrogram was similar to that of the electrocardiogram of the precordial lead; an initial downward deflection was followed by an upward deflection, the relative size 

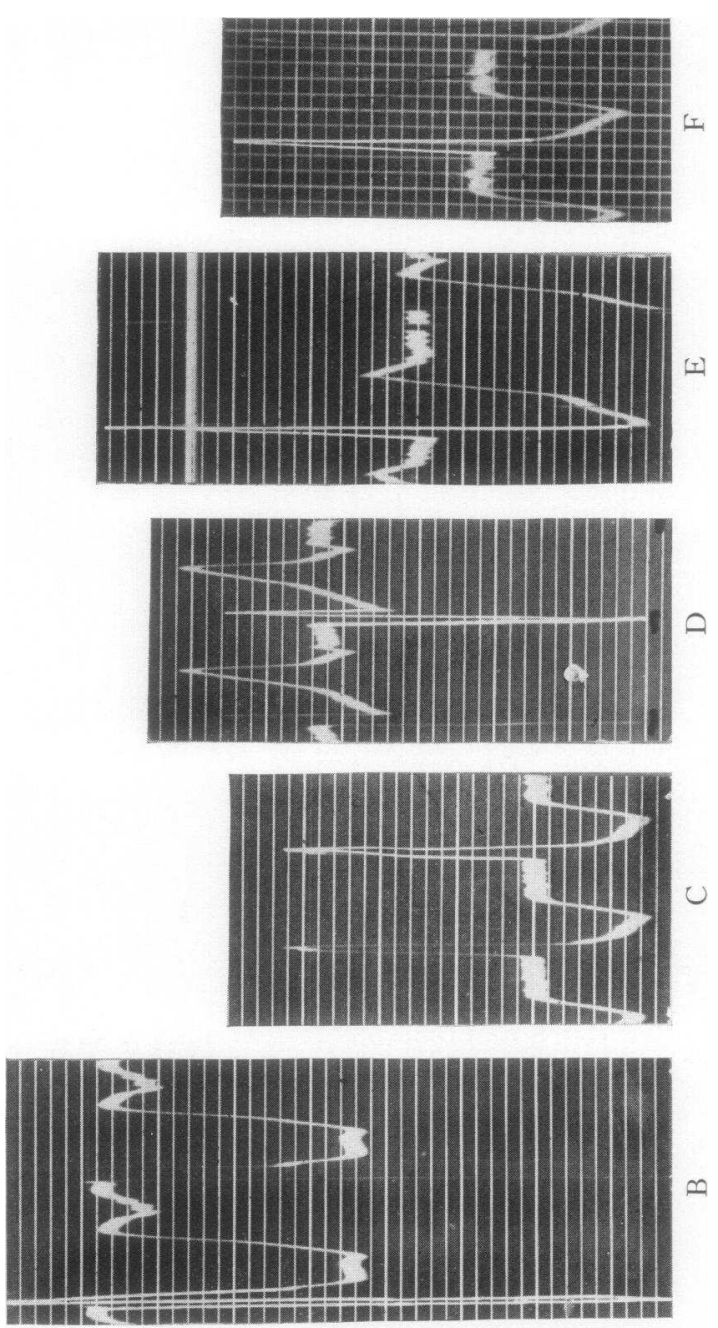

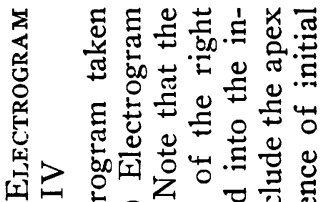

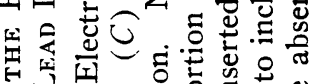

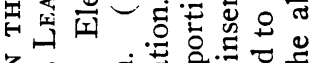

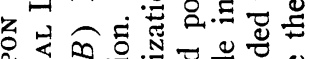

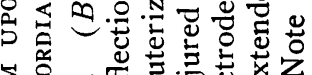

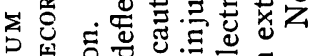

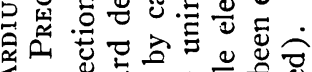

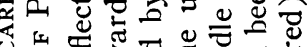

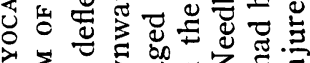

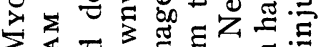

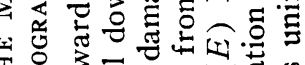

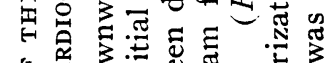

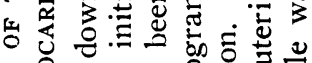
只 0

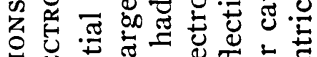
욜. 외 0 을

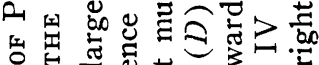

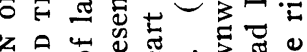

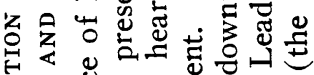

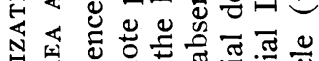

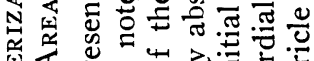

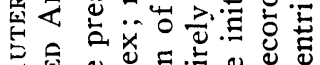

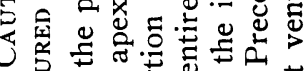

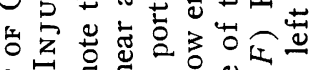

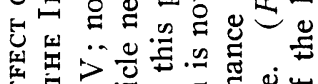

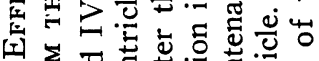

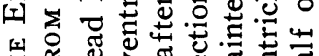

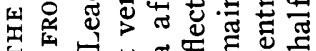

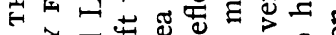

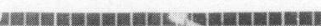

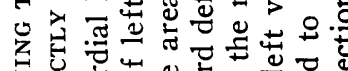

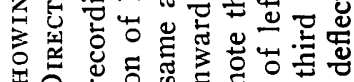

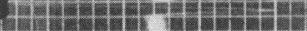

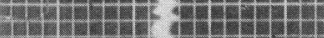

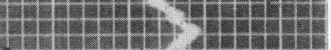
든 350

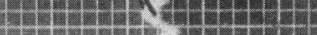

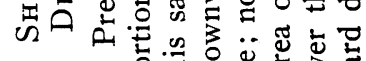

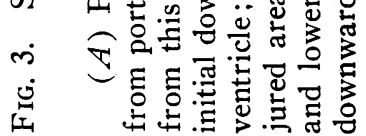


of which varied, depending upon the location of the anterior electrode. When the electrode was placed on the anterior surface of the left ventricle near the apex, the initial downward deflection was approximately equal to that of the upward deflection. ${ }^{2}$

Cauterization of this small area near the apex of the left ventricle resulted in a disappearance of the initial downward deflection in the electrogram taken from the electrode placed on the cauterized area, whereas a control electrogram taken immediately afterwards from an uninjured portion of the heart on the anterior surface of the right ventricle showed the initial downward deflection still preserved (Fig. 3). Needle electrodes inserted deep into the muscle below the cauterized area showed an absence of the initial downward deflection.

\section{Infarction of the posterior wall}

Ligation of the coronary arteries supplying the posterior wall or injury of this part of the myocardium by cauterization produced no change in the initial downward deflection. The only change observed was that the R-T interval was situated above the iso-electric line, originating from the downstroke before it reached the base line (Fig. 4). These findings are similar to those observed in man (11) (13).

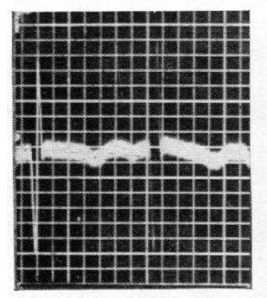

IV

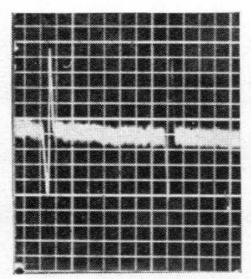

$\mathrm{V}$

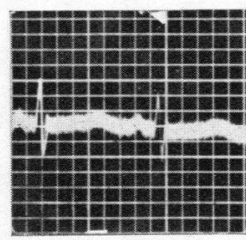

VI

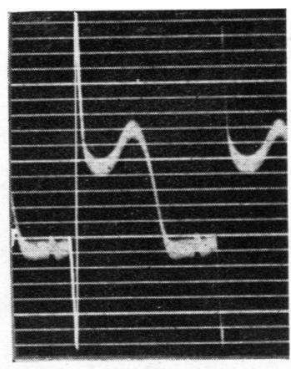

IV

B

Fig. 4. Showing the Effect of Injury to the Hfart Muscle on the Posterior Wall of the. Left Ventricle in Cat

( $A$ ) Precordial Leads IV, V, and VI before injury. Note the presence of initial downward deflection in Leads IV and V equal in amplitude to the upward deflection. $(B)$ Shows the effect in Lead IV of cauterization of the posterior wall of the left ventricle. Note that in $B$ there is no change in the initial downward deflection; the only alteration observed being that the $\mathrm{S}-\mathrm{T}$ interval comes off considerably above the isoelectric line and has a monophasic character.

2 In the electrogram with one electrode on the left ventricular surface and the other at some distant point the deflection obtained was approximately 20 times the value obtained in the indirect leads (5) ( 7 ). It was, therefore, necessary to tighten the string about 20 times the normal to obtain tracings with an amplitude approximating that usually seen in the indirect leads. In taking the electrogram from the surface of the right ventricle the string had to be tightened less than when the electrode was placed on the left ventricle, probably indicating that the voltage developed by the right ventricle is less than that developed by the left (4). 


\section{SURVIVAL EXPERIMENTS}

METHODS

In the chronic type of experiment the animals were operated upon under sodium amytal anesthesia with strict asepsis. A cannula was introduced through the mouth into the trachea and pulmonary inflation was maintained by means of a bellows. The chest was opened by an incision in the fourth interspace on the left sicle. By means of a self retaining retractor the fourth and fifth ribs were separated. The pericardium was longitudinally incised and drawn into the wound in the chest wall, thus preventing the expanding and contracting lungs from interfering during the exposure and ligation of the coronary artery. The artery of choice was dissected free from the epicardium and doubly ligated with silk ligatures. In all the survival experiments the anterior descending branch of the left coronary artery was the vessel ligated. The pericardium was then closed by interrupted sutures and the wound in the chest closed with the lungs in full expansion. Following the closure of the chest wall the tracheal cannula was removed. The animals were kept on the usual animal-house diet and allowed a minimum amount of exercise. Although the mortality was high we obtained five dogs which were followed for from 3 to 6 months after operation. Electrocardiograms were taken before operation, at frequent intervals after the ligation of the coronary artery during the first 24 hours, daily for the next 10 days, and weekly during the chronic stage.

In most instances where death occurred, it took place either immediately or soon after the ligation of the coronary artery. If the animal survived 24 hours the chances for its recovery were good. The animals that recovered appeared to be in good condition and about a week after operation acted like normal animals. One dog. Number 324, died three months after operation. The remaining four dogs were sacrificed four to six months after operation. We record also the results on one cat which died from pneumonia 36 hours after operation.

\section{Electrocardiogranis and pathologic findings}

Dog Number 261 (Fig. 5). The initial downward deflection originally prominent, had considerably diminished 7 hours after operation and was minimal from the $3 \mathrm{~d}$ to the 7 th day after operation. It tended thereafter to return gradually toward normal but never attained its preoperative amplitude. Forty-five days after operation the initial downward deflection was about one-half that of the upward deflection. The R-T interval changes resembled those observed in the human subject. The high amplitude of the (upright) T waves in Leads IV and V were practically identical with those observed in man and probably represent a sub-acute stage of infarction (13) (15). T waves of this type were observed only from the $3 d$ to the 5 th day after ligation of the coronary artery. After about the 9th day the electrocardiogram remained practically constant, and appeared to be that characteristic for the chronic stage.

This animal when sacrificed six months after operation showed dense pericardial adhesions on the anterior surface of the heart around the interventricular groove. An area of infarction about two centimeters in diameter involved chiefly the apex of the left ventricle, with beginning aneu- 
rysmal dilatation. The area of fibrosis was more marked on the endocardial than on the epicardial side of the left ventricle (Fig. 6).

Dog Number 297. The initial downward deflection was diminished somewhat four hours after ligation and was absent after 24 hours. It remained absent for 5 days and then gradually reappeared, but never returned to its preoperative value, and never exceeded about half the value

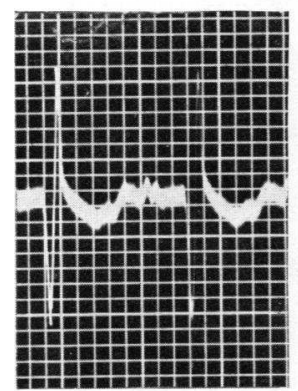

A

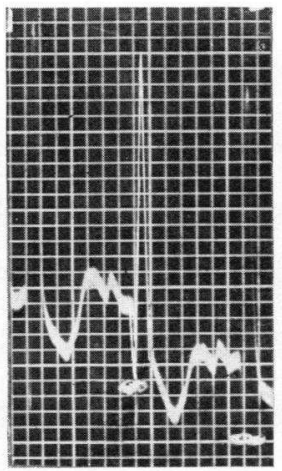

B

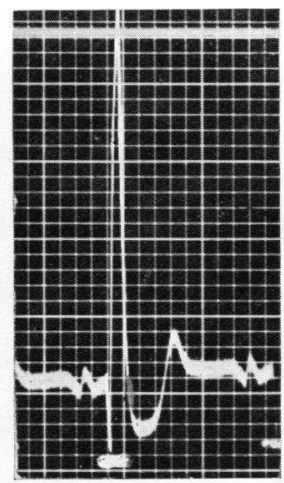

C

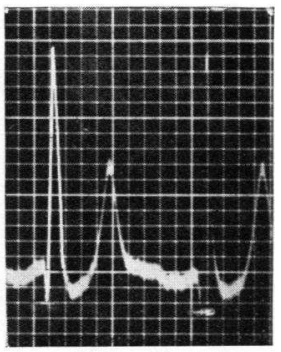

$\mathrm{D}$

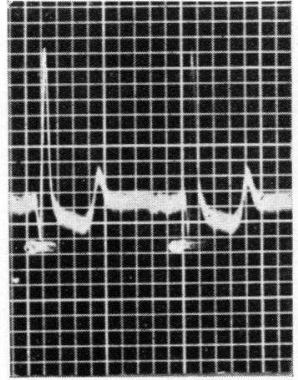

$\mathrm{E}$

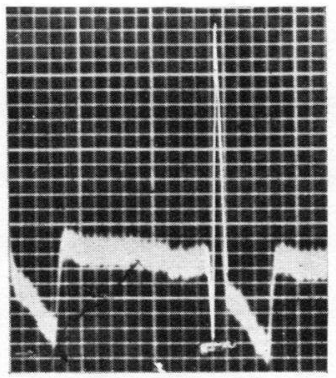

F

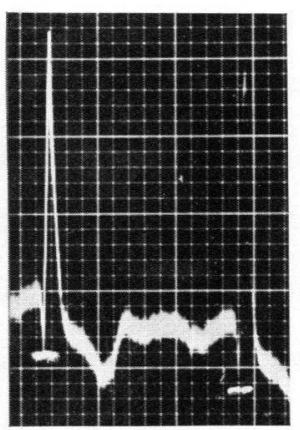

G

Fig. 5. Shows the Electrocardiograms from Precordial Lead IV from the Survival Experiment on Dog Number 261

$(A)$ before operation. (B) Seven hours after ligation of the anterior descending branch of left coronary artery. Note the marked diminution in the initial downward deflection and the slight depression of S-T interval. (C) 44 hours after operation. Note the diminution in the initial downward deflection and depression of S-T interval. (D) Three days after the operation; note the still further diminution in the initial downward deflection and the high amplitude of $\mathrm{T}$ waves referred to in the text. (E) 5 days after operation. Note the small amplitude of initial downward deflection and change in characteristics of the $\mathrm{T}$ wave. $(F) 11$ days after operation. The initial downward deflection has increased somewhat in amplitude and the S-T interval deviation has almost disappeared. (G) 6 months after operation (chronic stage). The initial downward deflection is small compared to the upward deflection. 
of the upward deflection. Tall upright $T$ waves were observed as a transient phenomenon from the $3 \mathrm{~d}$ to the 7 th day after operation.

This animal was sacrificed after 4 months, and showed the most extensive infarction observed in any of our experiments, involving the lower half of the anterior portion of the left ventricle including the apex and extending into the lateral walls.

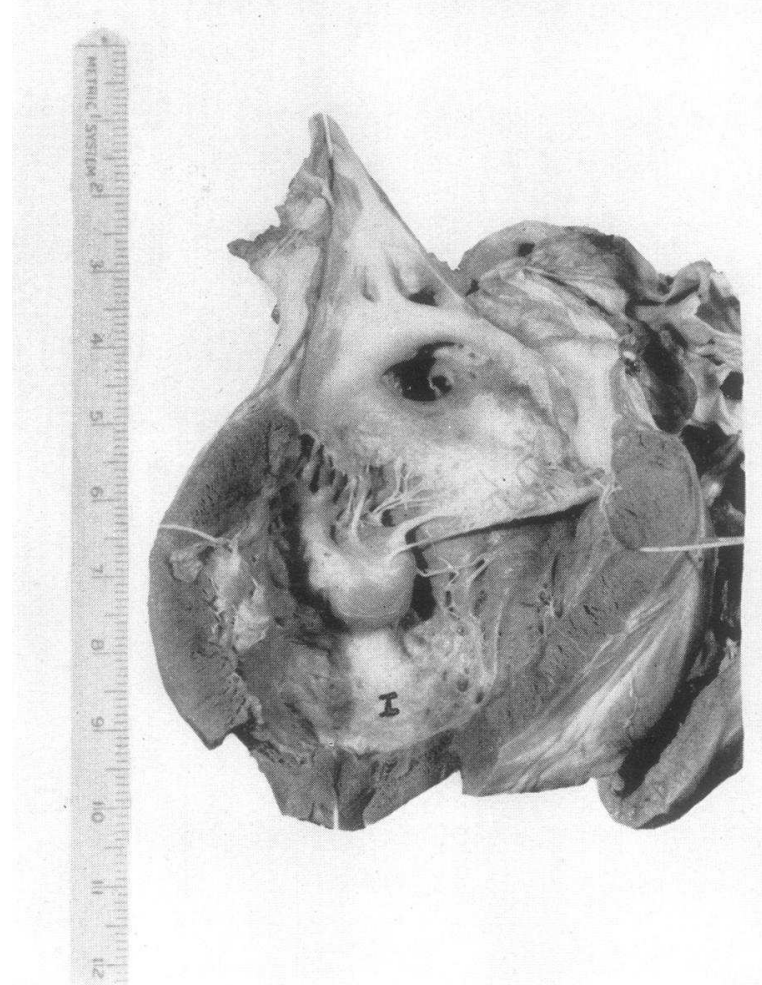

Fig. 6. Showing Heart of Dog Number 261, the Electrocardiograms of Which are Shown in Figure 5

This animal was sacrificed six months after operation. Note the area of chronic infarction involving the apex and lower third of anterior wall of left ventricle.

Dog Number 32t. The initial downward deflection was somewhat diminished after operation but was never abolished. Its amplitude remained about half that of the upward deflection except for one day (21st day after operation) when it exceeded the latter. On the 42d day after operation its amplitude was about one-third that of the upward deflection. The transient appearance of tall $\mathrm{T}$ waves was observed in this case also on the $3 d$ day following operation. 
This animal died of bronchopneumonia 3 months after operation. The heart showed moderate pericardial adhesions involving the anterior and lateral portion of the left ventricle. Upon injecting the coronary arteries with an opaque mass the left descending branch remained almost empty.

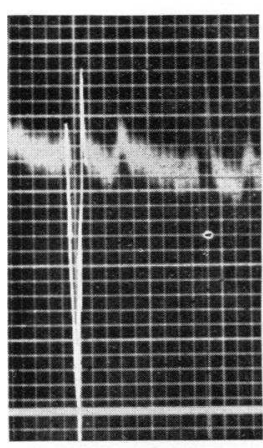

A

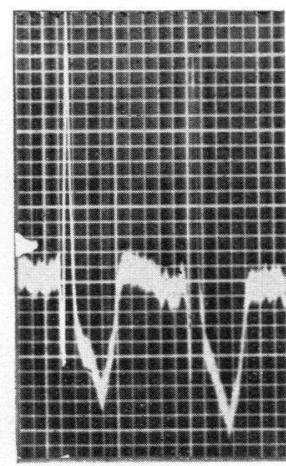

B

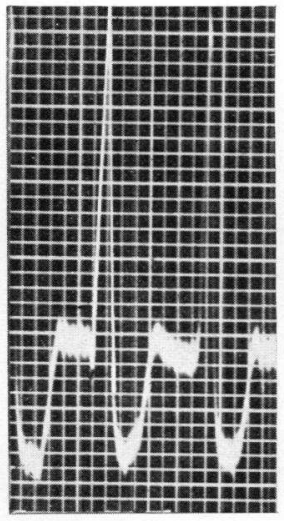

C

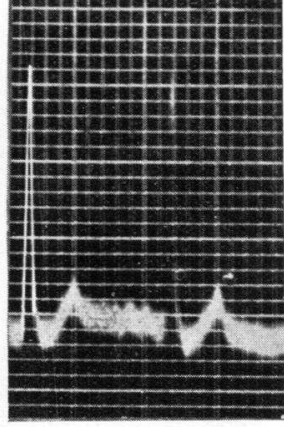

$\mathrm{D}$

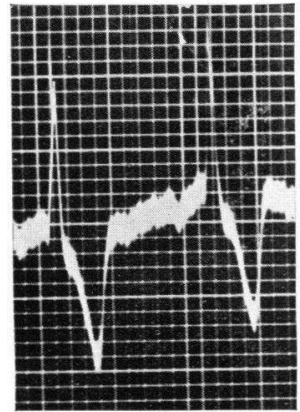

$\mathrm{E}$

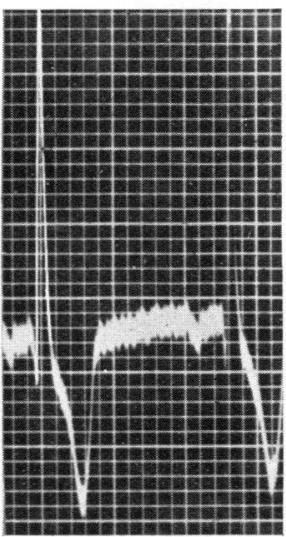

$\mathrm{F}$

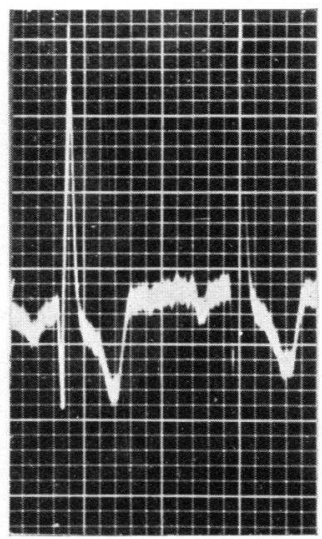

G

Fig. 7. Shows the Electrocardiograms from Precordial Lead IV from the Survival Experiment on Dog Number 328

$(A)$ Before operation. (B) 3 hours after ligation of the anterior descending branch of the left coronary artery. Note the marked diminution in the initial downward deflection and depression of the S-T interval. (C) 24 hours after operation. Note that the initial downward deflection is almost absent and that the S-T interval is situated below the iso-electric line. (D) 4 days after operation. Note the complete absence of initial downward deflection and the upright $\mathrm{T}$ wave. (E) 13 days after operation. Note the complete absence of initial downward deflection and the inverted $T$ wave. $(F) 27$ days after operation. Note the slight initial clownward deflection $(2 \mathrm{~mm}$.). (G) 6 months after operation. Note the increase in amplitude in the initial downward deflection now measuring $6 \mathrm{~mm}$. 
The slight amount of filling that resulted was due apparently to anastomosis from neighboring vessels. The area of infarction was small, involving only an area about two centimeters in diameter on the anterior surface of the left ventricle near the apex. There was no marked thinning of the left ventricular wall.

Dog Number 330. No definite diminution to the initial downward deflection occurred in the first 24 hours following operation. Thereafter, all the deflections were small but the initial downward deflection was especially reduced, being almost absent on the 5th day. By the 37th day the initial downward deflection had practically returned nearly to its preoperative amplitude.

This animal when sacrificed four months after operation showed only a small area of infarction about one and one-half centimeters in diameter involving the anterior portion of the left ventricle near the apex.

Dog Number 328 (Fig. 7). The initial downward deflection gradually disappeared following the operation and was almost absent 24 hours afterwards. It remained almost or entirely absent for about 14 days and then gradually reappeared. Six months after operation it had about one-third the amplitude of the upward deflection. Transient tall $\mathrm{T}$ waves were also observed in this animal.

This animal was sacrificed six months after operation. The cardiac findings were similar to those of Dog Number 261.

Cat Number 1. The electrocardiogram taken 135 minuies after ligation of the anterior lescending branch of the left coronary artery showed complete loss of the initial downward deflection in Leads IV and $\mathrm{V}$, and an R-T interval deviation situated below the iso-electric line. Subsequent tracings showed a return of the $\mathrm{R}-\mathrm{T}$ interval to the iso-electric line but the initial downward deflection was absent in the last tracing taken 28 hours after ligation.

The heart showed a marked area of softening involving the lower half of the anterior and lateral portions of the left ventricle.

\section{DISCUSSION}

Comparison of clectrocardiograms of precordial leads in man and in dogs and cats following coronary occlusion. The initial downward deflection from precordial leads, which in man is either markedly diminished or entirely absent after occlusion of the anterior descending branch of the left coronary artery, is preserved and undergoes almost no change in dogs and cats immediately following ligation of the anterior descending branch of the left coronary artery. Such a marked difference in response of the human subject and the $\log$ or cat invites explanation. To this end we have considered 3 possibilities as a cause for this variation: first, the difference in extent of the region supplied by the occluded vessel in each instance; second, the variation in time ensuing between the occlusion and the tak- 
ing of the tracing; and third, the change in the position of the left ventricle relative to the anterior electrode.

In human cases occlusion of the anterior descending branch of the left coronary artery usually occurs close to its point of origin; the resulting area of infarction, therefore, is usually the apex, adjacent lateral wall of the left ventricle and the lower anterior portion of the ventricular septum. The blood supply of the human heart differs considerably from that of the dog and cat. In man the anterior descending branch of the left coronary artery supplies an area corresponding to that which is supplied by the anterior descending, septal and a considerable portion of the circumflex branches of the left coronary artery in the dog and cat. Before comparing the electrocardiographic results in animals with those obtained in man, it is, therefore, important to reproduce as closely as possible in the experimental animal the location and relative size of the infarcted area.

From the standpoint of time, the electrocardiographic findings obtained immediately after experimental occlusion in animals cannot justly be compared with those of the human subjects, in whom, even under advantageous conditions, tracings are rarely taken until many hours after the occlusion has occurred.

Furthermore, since we have evidence that the initial downward deflection seen in the precordial leads is produced chiefly by the left ventricle (see page 740), we regard the position of the left ventricle in relation to the anterior chest wall as not unimportant. The fact that a considerably larger portion of the left ventricle presents anteriorly in the dog and cat than in man may account for the greater difficulty in causing, in the former, the disappearance of the initial downward deflection.

Disappearance of initial downward deflection in acute experiments the result of ligation of multiple coronary arteries and of extensive injury of heart muscle by cautcrization. The endeavor to cause infarction of an area of the dog's heart corresponding to that which results in the human heart after occlusion of the left descending branch at its origin was unsatisfactory for our purpose since dogs withstand multiple ligation of the large coronary arteries poorly, the animals usually dying within a few minutes after the last vessel has been tied. As already shown, we were unable during the short period of their survival to cause disappearance of the initial downward deflection in dogs by ligating multiple arteries. In cats, which withstand multiple ligation of coronary arteries relatively well, ligation of the anterior descending branch of the left coronary artery plus ligation of the anterior branch of the circumflex (together supplying the apex, anterior and lateral wall of the left ventricle and a portion of the ventricular septum) resulted in a disappearance of the initial downward deflection. This indicates that ultimate electrocardiographic changes depend upon the size and location of the infarct produced rather than upon the ligation of any particular vessel. 
Cauterization of the heart muscle in dogs and cats also resulted in a marked diminution or disappearance of the initial downward deflection. The surface which had to be cauterized, however, to bring about this result was relatively much more extensive in dogs than in cats. In the dog cauterization of the apex and lower part of the left ventricular wall, while interfering to some degree with the circulation of the underlying muscle, does not eliminate the possibility of the muscle of the damaged area receiving considerable nourishment from vessels in the neighborhood. In acute experiments it is apparently only when the circulation from these surrounding areas is also impaired that the initial downward deflection is either markedly diminished or entirely eliminated. Because of the smaller size of the cat's heart, cauterization, although not as extensive as in the dog with respect to area of surface, really involves a greater number of vessels and relatively greater depth of muscle.

In acute experiments the disappearance of the initial downward deflection was brought about in cats (1) after ligation of the anterior descending branch plus the anterior branch of the circumflex and (2) after cauterization of an extcnsiz' area of the anterior portion of the left ventricle. In dogs, the same effect was brought about only with the greatest difficulty. Ligation of vessels proved ineffectual; the only successful procedure was the cauterization of a massive portion of the heart muscle, relatively much more than is involved in the infarction observed in the human heart at necropsy.

In survival experiments, however, the initial downward deflection almost or entirely disappeared in three dogs and in one cat 24, 24, 48 and 2 hours respectively after ligation of the anterior descending branch of the left coronary artery. In two additional dogs moderate diminution of this deflection was produced. In the former group frequent electrocardiograms showed gradual decrease in size of the initial downward deflection beginning soon after operation, and progressing until it either finally disappeared or remained with an amplitude of only one to two mm. Only the left descending branch of the coronary artery was ligated in these experiments. The question arises as to why the disappearance of the initial downward deflection after ligation was gradual. We suggest that it was so because the maximum degree of cardiac damage or cardiac infarction accompanied by actual death of muscle probably developed only 24 to 48 hours after ligation of the anterior descending branch of the left coronary artery.

The observations in the survival experiments are quite similar to those in man if the time interval ensuing between occlusion and the taking of the electrocardiogram is taken into consideration. The records observed in the $\operatorname{logs} 24$ hours after ligation of the anterior descending branch of the left coronary artery are quite similar to those in man after occlusion of this vessel; in fact, it is almost impossible to distinguish typical records. In 
most instances, the disappearance of the initial downward deflection is not as complete in the dog as in man. The tracing taken 24 to 48 hours after ligation in the dog often shows a slight downstroke of 1 to $2 \mathrm{~mm}$. preceding the upstroke, whereas in man the downstroke is in most instances entirely absent at a similar interval following occlusion.

The return of the initial downward deflection more completely and more rapidly in dog than in man may be explained in several ways: (a) that a relatively smaller vessel was occluded in the dog than occurs in patients and that the resulting infarct was, therefore, relatively smaller; $(b)$ that in the dog, the remaining vessels were normal and were, therefore, capable of furnishing the maximum of collateral circulation, whereas in patients marked atheroma is often present in the other coronary arteries; $(c)$ that in the dog, the vessel distal to the ligation being normal may still function to some degree with the development of anastomoses, whereas in patients frequently the presence of atheroma of the vessels distal to the point of thrombosis with consequent slowing of the blood current may lead to occlusion below the point of initial thrombosis.

The presence of the high amplitude of the $T$ wave in precordial leads which was observed as a transient phenomenon in all of our dogs in the subacute stage of infarction is almost identical with that observed in man (13) (15). In the latter it has been observed as long as 2 to 4 weeks after the onset of the acute infarction. Its presence is of considerable importance. Bohning and Katz (2) have recently reported the presence of such $\mathrm{T}$ waves in human indirect leads; and Wood and Wolferth have reported their presence in precordial leads (15).

Cause of production and disappearance of initial downward deflection of precordial leads. The configuration of the electrogram from the ventricular surface and of the electrocardiogram from the precordial leads is quite similar (7); the higher voltage in the former is due the electrode being placed directly upon the heart, whereas in the latter, it is placed at some distance from the heart. The electrogram, when the electrode on the heart is placed over the normal muscle, shows an initial downward deflection diminishing as the great vessels are approached (5) (10). This relation also holds for the electrocardiograms from the precordial leads. From these findings it would appear that when in the precordial leads a large electrode is used (i.e., one covering the entire precordium), the electrocardiogram may be considered as due to the summation of the action currents from the muscles of the entire heart; where a small electrode is used the electrocardiogram is produced chiefly by action currents from the muscle lying immediately beneath the electrode and only to a lesser degree by those from muscle more distant. Since the voltage recorded is probably much greater from the left ventricle than from the right it is probably the former which largely determines the ventricular complex in the precordial leads. For example in the experiment (Fig. 3) in which the apex and 
lower third to half of the anterior wall of the left ventricle was cauterized and the electrogram from the cauterized area showed a disappearance of the initial downward deflection formerly present, the initial downward deflection was preserved in the electrogram of the uninjured muscle of the right ventricle. The precordial lead (Lead IV), using a large electrode covering the entire precordium, showed a complete absence of the initial downward deflection. The same was true in survival experiments in the dog and in human cases in which the infarction involved only the lower anterior half of the left ventricle while the right ventricle was intact.

From these data it would appear that we have information regarding the portions of the heart responsible for determining the presence or absence of the initial downward deflection in precordial leads. The important area appears to be the apex, lower anterior and lateral walls of the left ventricle and probably in addition the lower anterior and left side of the interventricular septum. ${ }^{3}$ The chief evidence that we have obtained supporting this statement is: (1) the absence of the initial downward deflection from precordial leads in 12 human subjects in whom infarction had involved this area; and (2) the disappearance of the initial downward deflection after experimental ligation of vessels that supply this area or after cauterization of the heart muscle of this region. ${ }^{4}$

\section{SUMMARY AND CONCLUSIONS}

In acute experiments upon dogs and cats ligation of the anterior descending branch of the left coronary artery, while causing R-T deviation in precordial leads produced practically no change in the initial downward deflection of the ventricular complex. In general, ischemia or damage to a much greater portion of the myocardium than that supplied by the anterior descending branch of the left coronary artery was required to produce a marked diminution or disappearance of the initial downward deflection. Diminution or disappearance of this deflection was produced in cats after ligation of the anterior branch of the left coronary plus the anterior branch of the circumflex arteries; or in dogs and cats after cauterization of a considerable portion of the surface of the cardiac muscle.

${ }^{3}$ In the human cases, infarction of the lower anterior portion of the septum is nearly always present after occlusion of the left descending branch; in dogs, involvement of the septum does not occur unless the septal branch is ligated in addition to the anterior descending branch of the left coronary artery.

4 Wilson (11) has pointed out the similarity of the electrocardiogram from precordial leads in cases of infarction of the anterior surface of the left ventricle to the electrogram obtained when the anterior electrode is placed at the pulmonic area just above the pulmonary valves or at other points that lie opposite one of the valvular orifices. He suggests that the early occurrence of a prominent upstroke not preceded by a preliminary downstroke in these curves may be due to the presence of a large infarct which, being composed of dead and inactive muscle, has an effect upon the electrocardiogram similar to that which might be produced by a window or orifice in the anterior wall of the heart. 
In survival experiments marked diminution or disappearance of the initial downward deflection could be produced by ligation of the anterior descending branch of the left coronary artery alone. In 3 out of 5 dogs this deflection almost completely disappeared 24 to 48 hours after ligation. In one cat it disappeared 2 hours after operation. In one dog it was markedly diminished on the 5th day after operation and in another moderately diminished on the $3 \mathrm{~d}$ day. In all 5 dogs, later during the recovery stage, it increased again; in one instance the return was complete, in the remaining 4, the deflection regained one-fourth to one-half its original amplitude. In man the initial downward deflection which disappears during the acute stage of infarction usually does not return in the chronic stage; occasionally, however, a partial return occurs. The return of this deflection in the dog is probably to be explained by the smaller relative size of the area involved by the chronic infarct and by the larger portion of the left ventricle in relation with the anterior chest wall in the dog.

Extremely tall $\mathrm{T}$ waves observed in these experiments as a transient phenomenon are believed to characterize a subacute stage of infarction.

Ligation of the coronary arteries supplying the posterior wall of the left ventricles or injury of this area by cauterization produced R-T interval deviations above the iso-electric line but no change in the amplitude of the initial downward deflection.

\section{BIBLIOGRAPHY}

1. Barnes, A. R., and Whitten, M. B., Study of the R-T interval in myocardial infarction. Am. Heart J., 1929, 5, 142.

2. Bohning, A., and Katz, L. N., Unusual changes in the electrocardiogram of patients with recent coronary occlusion. Am. J. M. Sc., 1933, 186, 39.

3. Crawford, J. H., Roberts, G. H., Abramson, D. I., and Cardwell, J. C., Localization of experimental ventricular myocardial lesions by the electrocardiogram. Am. Heart J., 1932, 7, 627.

4. Hermann, G. R., and Wilson, F. N., Ventricular hypertrophy. A comparison of electrocardiographic and postmortem observations. Heart, 1921, 9, 91.

5. Lewis, T., and Rothschild, M. A., The excitatory process in the dog's heart. Part II. The ventricles. Phil. Trans. Royal Soc., 1915, 206, 181.

6. Otto, H. L., The effect of obstruction of coronary arteries upon the T-wave of the electrocardiogram. Am. Heart J., 1929, 4, 346.

7. Wilson, F. N., The distribution of the potential differences produced by the heart beat within the body and at its surface. Am. Heart J., 1930, 5, 599.

8. Wilson, F. N., Johnston, F. D., Hill, I. G. W., and Graut, G. C., Form of electrocardiogram in experimental myocardial infarction. Proc. Soc. Exper. Biol. and Med., 1932, 30, 799.

9. Wilson, F. N., Barker, P. S., MacLeod, A. G., and Klostermeyer, L. L., The electrocardiogram in coronary thrombosis. Proc. Soc. Exper. Biol. and Med., 1932, 29, 1006.

10. Wilson, F. N., MacLeod, A. G., and Barker, P. S., The order of ventricular excitation in human bundle branch block. Am. Heart J., 1932, 7, 305. 
11. Wilson, F. N., MacLeod, A. G., Barker, P. S., Johnston, F. D., and Klostermeyer, L. L., The electrocardiogram in myocardial infarction with particular reference to the initial deflections of the ventricular complex. Heart, 1933, 16, 155.

12. Wolferth, C. C., and Wood, F. C., The electrocardiographic diagnosis of coronary occlusion by the use of chest leads. Am. J. M. Sc., 1932, 183, 30 .

13. Wood, F. C., Bellet, S., McMillan, T. M., and Wolferth, C. C., Further observations on the use of direct chest leads in coronary occlusion. Arch. Int. Med., 1933, 52, 752.

14. Wood, F. C., and Wolferth, C. C., Experimental coronary occlusion. Inadequacy of the three conventional leads for recording characteristic action current, in certain sections of the myocardium: An electrocardiographic study. Arch. Int. Med., 1933, 51, 771.

15. Wood, F. C., and Wolferth, C. C., Huge T waves in precordial leads in cardiac infarction. Am. Heart J. (In press.) 